03.3

\title{
Трехмерная структура течения в окрестности импульсного поверхностного дугового разряда в магнитном поле
}

\author{
() П.Н. Казанский ${ }^{1}$, А.Я. Котвицкий ${ }^{1,2}$, И.А. Моралев ${ }^{1}$ \\ ${ }^{1}$ Объединенный институт высоких температур РАН, Москва, Россия \\ ${ }^{2}$ Московский государственный технический университет им. Н.Э. Баумана, Москва, Россия \\ E-mail: fokkoo@yandex.ru
}

Поступило в Редакцию 25 ноября 2020г.

В окончательной редакции 3 февраля 2021 г.

Принято к публикации 25 февраля 2021 г.

\begin{abstract}
Описана структура течения, возникающая при движении импульсного поверхностного дугового разряда в поперечном магнитном поле. Показано, что движение плазменного канала приводит к формированию тороидального вихря. При этом характерная скорость подсоса газа к стенке модели в аэродинамическом следе дуги составляла $30-50 \mathrm{~m} / \mathrm{s}$ и соответствовала величине до $40 \%$ от максимальной скорости расширения газа в фазе нагружения.
\end{abstract}

Ключевые слова: плазменный актуатор, магнитная гидродинамика, дуговой разряд.

DOI: 10.21883/PJTF.2021.11.50998.18631

Электрические дуговые разряды в магнитном поле довольно часто используются в различных технических приложениях: плазмотронах [1,2], дуговых размыкателях [3], плазменных актуаторах [4-7]. Бо́льшая часть исследований в этой области сфокусирована на параметрах плазмы дугового канала и приэлектродных явлениях. Структуре течения в окрестности плазменного канала уделяется гораздо меньше внимания, при этом именно она отвечает за теплообмен между дугой и внешним газом, а также за индуцированный магнитогидродинамическим (МГД) взаимодействием конвективный перенос во внешней среде.

Существующая литература по структуре течения в окрестности дуги посвящена стабилизированным (между силой Ампера и силой аэродинамического сопротивления) дугам, для которых расчеты принципиальной структуры течения [8,9] проведены еще в 80-х годах прошлого века. Известно, что благодаря тому, что сила Ампера не потенциальна, на границе проводящей области формируется пара вихрей $[8,10]$. Обтекание этой замкнутой циркуляционной области вместе с каналом дуги приводит к возникновению резкой границы между набегающим потоком и нагретым газом [9] и позволяет использовать „аэродинамические“ модели дугового канала [11]. Экспериментальные исследования структуры течения, подтверждающие эти расчеты, также проводились для стационарной конфигурации, при этом структура течения восстанавливалась по распределению температуры в дуговом канале $[12,13]$. Прямые измерения структуры течения до последнего времени отсутствовали.

Структура течения в окрестности импульсного дугового разряда в магнитном поле исследована экспериментально и теоретически в [14] в рамках двумерного подхода. Показано, что за стадией теплового расшире- ния, вызванной импульсным энергоподводом в разряд, формируется течение, аналогичное случаю стабилизированной дуги. При этом дуговой канал вместе с парой ассоциированных вихрей и границей дуговой каверны движется в направлении силы Ампера. Структура течения в этот момент времени определяется как тепловым расширением газа, так и действием пондеромоторной силы. После окончания импульса тока происходит релаксация распределения давления с формированием асимметричной волны разрежения. В области взаимодействия остается вихрь, который обеспечивает достаточно длительное (до единиц $\mathrm{ms}$ ) перемешивание среды со скоростями в десятки $\mathrm{m} / \mathrm{s}$ и теоретически может быть использован для управления смешением или отрывом потока.

На данный момент за рамками теоретических и экспериментальных исследований осталось влияние конечной длины проводящего канала. При достаточно больших задержках, когда расстояние, на которое выносится дуга, становится сопоставимым с межэлектродным, течение становится трехмерным. Этот фактор, очевидно, будет оказывать существенное влияние на структуру вихревых возмущений, созданных импульсной дугой.

Целью настоящей работы является экспериментальное исследование трехмерной структуры течения вблизи импульсного дугового разряда в магнитном поле.

Схема эксперимента приведена на рис. 1,a. Эксперименты проводились в безвихревой камере размером $400 \times 400 \times 400 \mathrm{~mm}$. Дуговой разряд формировался между парой тонких вольфрамовых электродов, расположенных на поверхности керамической пластины. Расстояние между электродами $z_{\text {gap }}=7 \mathrm{~mm}$, разряд инициируется между парой треугольных обострителей, разделенных промежутком в $1 \mathrm{~mm}$. 

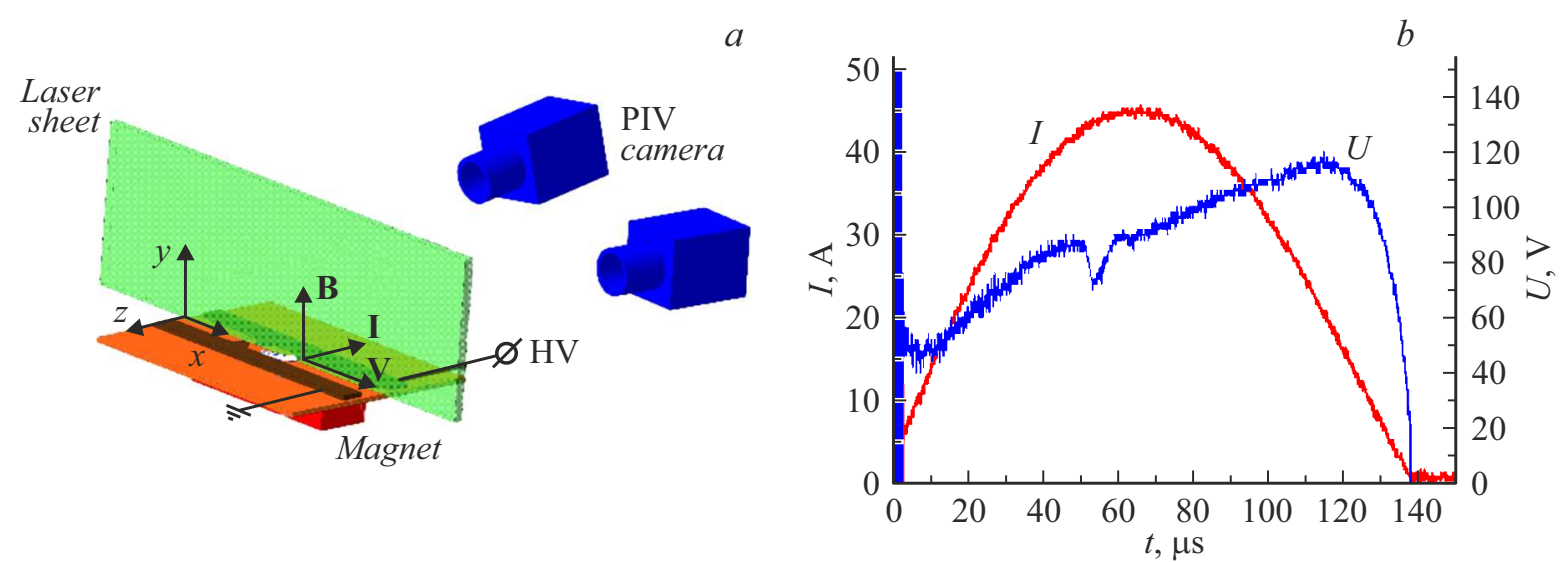

Рис. 1. Схема измерения поля скоростей методом PIV вблизи поверхностного дугового разряда в магнитном поле $(a)$, а также типичная осциллограмма тока и напряжения плазменного актуатора $(b)$.

Дуга питалась импульсом тока синусоидальной формы длительностью $130 \mu$ s и амплитудой 47 А (рис. $1, b$ ). По мере движения плазменного шнура вдоль обострителей рельсовых электродов длина шнура росла, что в конечном счете приводило к некоторому росту напряжения на электродах (рис. $1, b$ ). Разрядный промежуток помещался в однородное магнитное поле с индукцией $0.2 \mathrm{~T}$, направленное перпендикулярно поверхности пластины.

Поле скоростей исследовалось с помощью системы Stereo PIV LaVision FlowMaster. Поток засеивался частицами масла диаметром $\sim 1 \mu \mathrm{m}$ с временем динамической релаксации порядка $1-2 \mu$ s. Частицы подсвечивались двумя последовательными лазерными импульсами, сформированными в лазерный нож толщиной $0.5 \mathrm{~mm}$, задержка между которыми составляла $\sim 2 \mu$ s. Изображения регистрировались с помощью пары камер с разрешением 4 Мріх, установленных под углом $20^{\circ}$ друг к другу. Камеры были укомплектованы объективами MicroNikkor f 105 с удлиненным задним рабочим отрезком и Шаймпфлюг-адаптерами. Обработка изображений велась кросс-корреляционным методом с размером окна $32 \times 32$ ріх и перекрытием $50 \%$. Результирующее разрешение векторных полей составило $0.2 \mathrm{~mm}$ в плоскости лазерного ножа. Для получения трехмерного поля скорости снималось 30 двумерных распределений компонент $\left(v_{x}, v_{y}, v_{z}\right)$, отстоящих друг от друга на расстояние $\Delta z=1 \mathrm{~mm}$. Результирующее поле скоростей получалось при осреднении 70 мгновенных кадров.

Исследование структуры течения в окрестности дуги методом PIV (Particle Image Velocimetry) впервые позволило получить трехмерную картину течения в окрестности дуги (рис. 2). В момент пробоя в межэлектродном промежутке происходит формирование токопроводящего канала, сопровождающееся распространением в окружающем разряд газе цилиндрической ударной волны, которая достаточно быстро диссипирует в сферическую волну сжатия-разрежения. Аналогичные

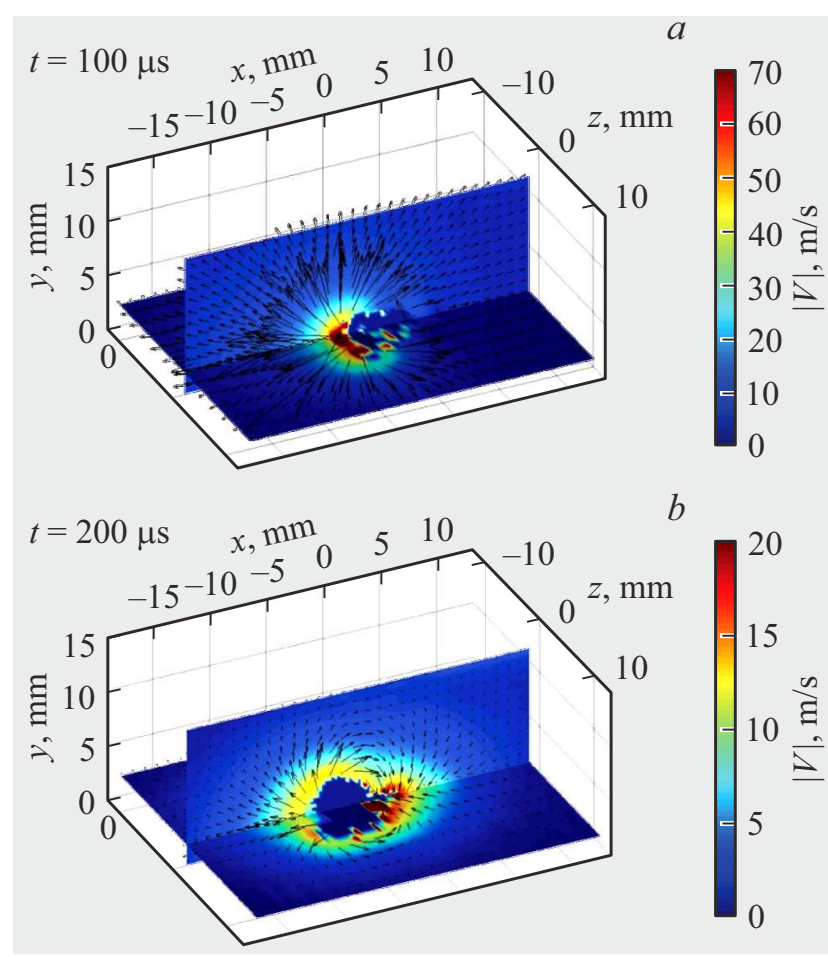

Рис. 2. Трехмерные поля скоростей течения, индуцированного приповерхностной дугой во внешнем магнитном поле.

структуры наблюдались в работе [15]. После ухода волны у поверхности пластины остается горячая область с низкой плотностью. Продолжающийся джоулев нагрев приводит к практически симметричному расширению тепловой каверны (рис. 2,a), что объясняется почти тридцатикратной разностью между работой теплового расширения и величиной пондеромоторной силы для рассматриваемых параметров импульса.

В результате действия силы Ампера расширение дуговой каверны со временем все же становится асим- 

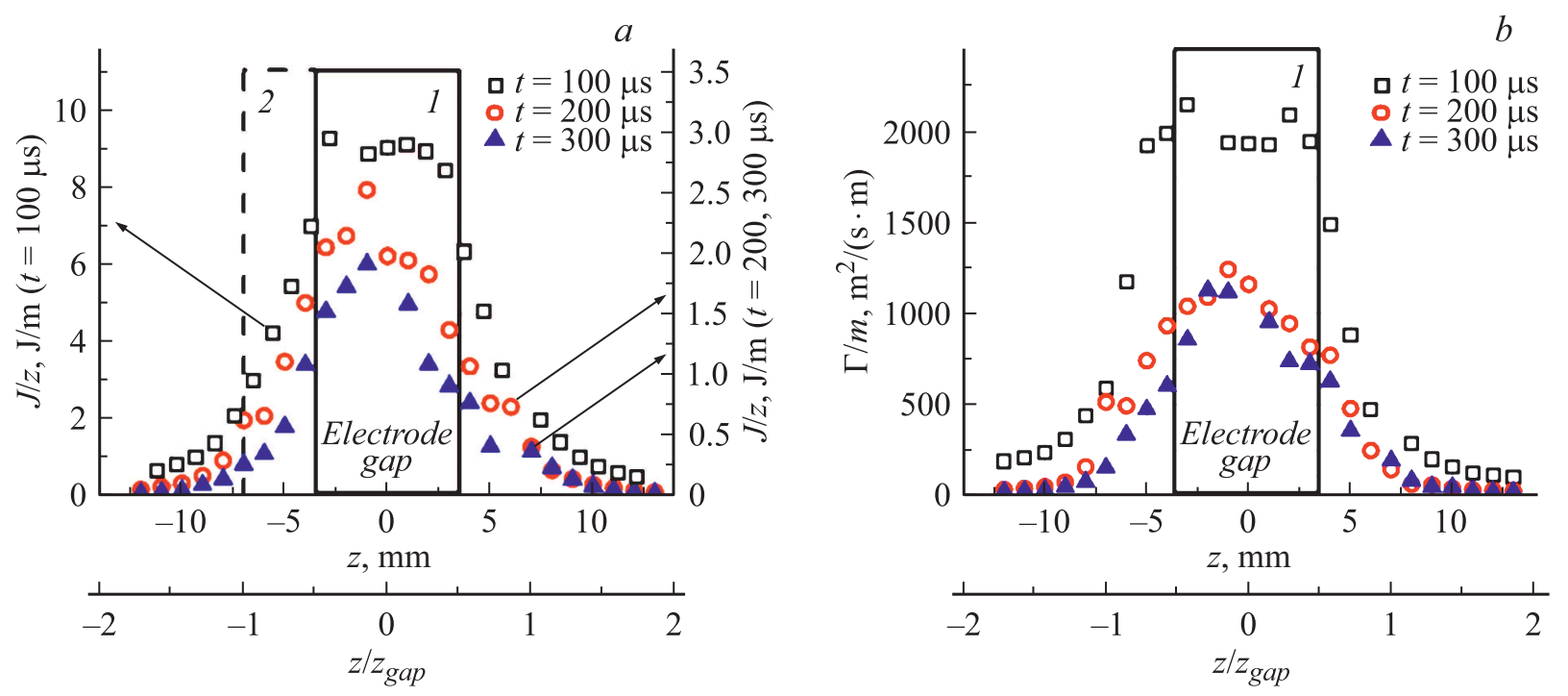

Рис. 3. Зависимость линейной плотности кинетической энергии газа $(a)$ и циркуляции $(b)$ в разных сечениях трехмерной структуры возмущения.

метричным. В окрестности дугового канала формируется вытянутая пара противоположно вращающихся вихрей, которые замыкаются в приэлектродных областях. По завершении токового импульса от каверны распространяется волна разрежения, которая уносит с собой сферически-симметричную часть импульса. В области взаимодействия остается асимметричное вихревое кольцо (рис. $2, b$ ), в донной части которого наблюдается подcoc газа к обтекаемой стенке. Максимальная скорость подсоса газа может достигать $30-50 \mathrm{~m} / \mathrm{s}$ и составляет до $40 \%$ от максимальной скорости расширения газа (или движения дуги) в момент энергоподвода.

Для оценки степени трехмерности течения были использованы кинетическая энергия возмущения $J$

$$
J=\sum_{i} \sum_{j} \frac{\rho \Delta V\left(v_{x}^{2}+v_{y}^{2}\right)_{i j}}{2}
$$

и $z$-компонента циркуляции поля скоростей $\Gamma_{z}$

$$
\Gamma_{z}=\sum_{i} \sum_{j}\left(\frac{\partial v_{y}}{\partial x}-\frac{\partial v_{x}}{\partial y}\right)_{i j}
$$

полученные в различных плоскостях, нормальных токовому каналу. Плотность засеянной области воздуха $\rho$ в рассматриваемом элементарном объеме $\Delta V$ принималась равной $1.3 \mathrm{~kg} / \mathrm{m}^{3}$.

Результаты таких интегральных оценок для задержек в 100, 200 и $300 \mu$ s представлены на рис. 3. Максимум кинетической энергии газа находится в плоскости симметрии электродного промежутка. В момент времени $100 \mu \mathrm{s}$ после пробоя линейная плотность кинетической энергии холодного газа одинакова во всем межэлектродном промежутке (область 1 ) и составляет $10 \mathrm{~J} / \mathrm{m}$. Скорость расширения быстро падает вне токового канала в области $2\left(z / z_{\text {gap }}\right.$ от -1 до -0.5$)$ и симметричной области $z / z_{\text {gap }}$ от 0.5 до 1 . Несмотря на доминирующую роль теплового расширения в формировании профиля скоростей на стадии энерговклада, циркуляция в рассматриваемой области отлична от нуля (рис. $3, b$ ). Поскольку характерная длина дуги на данном этапе больше характерного диаметра тепловой каверны, циркуляция оказывается также сосредоточена в межэлектродном промежутке.

При больших задержках происходит увеличение высоты каверны и формирование вихревого кольца. Через $200 \mu \mathrm{s}$ после пробоя линейная плотность кинетической энергии падает в плоскости оси симметрии модели до $2.5 \mathrm{~J} / \mathrm{m}$, что объясняется уносом энергии волной разгрузки в конце импульса тока.

Таким образом, исследована трехмерная структура течения, индуцированного импульсным дуговым разрядом конечной длины в поперечном магнитном поле. Показано, что в результате МГД-взаимодействия формируется деформированный тороидальный вихрь, характерные скорости в котором достигают 20-40\% от скорости передней границы каверны (или самого дугового канала) в активной части импульса.

\section{Финансирование работы}

Работа выполнена при финансовой поддержке Российского научного фонда (грант 18-19-00740).

\section{Конфликт интересов}

Авторы заявляют, что у них нет конфликта интересов. 


\section{Список литературы}

[1] H. Saito, H. Matsumoto, T. Fujino, J. Therm. Spray Technol., 29 (3), 333 (2020). DOI: 10.1007/s11666-020-00983-1

[2] I.B. Klementyeva, V.A. Biturin, B.N. Tolkunov, I.A. Moralev, High Temp., 49 (6), 788 (2011). DOI: $10.1134 / \mathrm{S} 0018151 \mathrm{X} 11060149$

[3] R. Ma, M. Rong, F. Yang, Y. Wu, H. Sun, D. Yuan, H. Wang, C. Niu, IEEE Trans. Plasma Sci., 41 (9), 2551 (2013). DOI: $10.1109 /$ TPS.2013.2273832

[4] Д.С. Мунхоз, В.А. Битюрин, А.И. Климов, П.Н. Казанский, И.А. Моралев, Л.Б. Поляков, Б.Н. Толкунов, ЖТФ, 87 (7), 997 (2017). DOI: 10.21883/PJTF.2021.11.50998.18631 [Пер. версия: $10.1134 / \mathrm{S} 1063784217070155]$.

[5] P.N. Kazanskiy, I.A. Moralev, V.A. Bityurin, A.V. Efimov, J. Phys.: Conf. Ser., 774 (1), 012153 (2016). DOI: $10.1088 / 1742-6596 / 774 / 1 / 012153$

[6] B. Pafford, Y.-J. Choi, J. Sirohi, L.L. Raja, in 44th AIAA Plasmadynamics and Lasers Conf. (San Diego, CA, 2013), AIAA 2013-2886. DOI: 10.2514/6.2013-2886

[7] S.B. Leonov, I.V. Adamovich, V.R. Soloviev, Plasma Sources Sci. Technol., 25 (6), 63001 (2016). DOI: $10.1088 / 0963-0252 / 25 / 6 / 063001$

[8] M.D. Cowley, A boundary-layer model for balanced arcs (Cambridge, MA, 1967).

[9] W.T. Lord, J. Fluid Mech, 35 (4), 689 (1969). DOI: $10.1017 / \mathrm{S} 0022112069001388$

[10] V. Bityurin, A. Bocharov, in 39th Aerospace Sciences Meeting and Exhibit (American Institute of Aeronautics and Astronautics, 2001), AIAA-2001-0793.

DOI: $10.2514 / 6.2001-793$

[11] T.W. Myers, W.C. Roman, AIAA J., 5 (11), 2011 (1967). DOI: $10.2514 / 3.4354$

[12] N. Sebald, Appl. Phys., 21 (3), 221 (1980). DOI: $10.1007 / \mathrm{BF} 00886172$

[13] Z. Guo, Int. J. Heat Mass Transf., 27 (3), 383 (1984). https://doi.org/10.1016/0017-9310(84)90285-0

[14] I. Moralev, P. Kazanskii, V. Bityurin, A. Bocharov, A. Firsov, E. Dolgov, S. Leonov, J. Phys. D: Appl. Phys., 53 (42), 425203 (2020). DOI: 10.1088/1361-6463/ab9d5a

[15] Ф.Н. Глазырин, И.А. Знаменская, И.В. Мурсенкова, Д.С. Наумов, Н.Н. Сысоев, Письма в ЖТФ, 42 (2), 16 (2016). [Пер. версия: 10.1134/S1063785016010223]. 